\title{
Three-dimensional analysis of gait in children and adolescents with juvenile idiopathic arthritis
}

\author{
Katarzyna BaZARniK-Mucha, SŁaWOMir Snela, Magdalena SZCZEPANiK, \\ AgNIESZKA JARMUZIEWICZ, AGNIESZKA GUZIK, OlgA WOLIŃSKA, MARIUSZ DRUŻBICKI* \\ Faculty of Medicine, University of Rzeszów, Rzeszów, Poland.
}

\begin{abstract}
Purpose: The paper aimed to assess the gait pattern in children and adolescents with juvenile idiopathic arthritis (JIA) treated at the rehabilitation center and to assess changes in this pattern after the end of treatment and 9 months later. Methods: 50 children with JIA were enrolled into the study. 35 healthy volunteers were enrolled into the study for a comparison. Spatiotemporal and kinematic gait parameters were obtained using a movement analysis system. The Gait Deviation Index (GDI) was calculated. The assessment was performed three times: on the day of admission to the rehabilitation center, after the end of a 4-week treatment period and 9 months later. Results: With regard to the majority of spatiotemporal and kinematic parameters, differences in their distribution were highly statistically significant between the study group and the control group $(p<0.001)$. In two subsequent tests, differences were less significant when compared to the control group $(p<0.01)$. In the study group, ranges of motion in the sagittal plane in the hip $(p<0.01)$, knee $(p<0.001)$ and ankle joints $(p<0.01)$ increased significantly between tests 1 and 2 , and 1 and 3 . A significantly lower value of GDI was observed in the study group (right limb; $p=0.036$ ). Conclusions: The gait pattern of children with JIA is significantly different from the one observed in healthy children. A rehabilitation program significantly improved gait in children with JIA, but differences compared to healthy children were still observed, and it indicates that the abnormal gait pattern became permanent in this group of subjects.
\end{abstract}

Key words: rehabilitation, arthritis, juvenile, 3D gait analysis

\section{Introduction}

Juvenile idiopathic arthritis (JIA) is the most common chronic juvenile arthropathy and its etiology has not been fully known. It is a group of diseases of a chronic character, with symptoms appearing before the age of 16 [17]. Treatment is aimed to minimize or inhibit inflammatory and immune activity of the disease and to prevent from other complications. Rehabilitation and appropriate physical activity are of vital significance with regard to functioning of the motor system, apart from routine and appropriately tailored pharmacotherapy. Taking into account new and more effective medicines and complex therapeutic management in treatment of children with JIA, one should ask about their efficacy in relation to maintaining motor skills [2], [22].

The gait pattern in children and adolescents with JIA may show subtle compensatory changes in response to arthralgia and limb deformations. Regarding more severe forms and periods of exacerbation complex gait abnormalities may appear, and, in many cases, they are difficult to be assessed clinically [6], [10], [11], [17]. The general condition of a child with chronic arthritis affects gait abnormalities. Patients tend to walk on the lateral foot border with limited loading of the medial metatarsal heads, hallux, and lesser toes. There are abnormalities with regard to kinematic, kinetic and spatiotemporal parameters. Increased hip flexion, limited knee extension at terminal stance and reduced ankle plantar flexion are reported [10]. Fairbum et al.

\footnotetext{
* Corresponding author: Mariusz Drużbicki, Faculty of Medicine, University of Rzeszów, al. mjr. W. Kopisto 2a, 35-310, Rzeszów, Poland. E-mail: mdruzb@ur.edu.pl

Received: November 24th, 2019

Accepted for publication: March 9th, 2020
} 
[10] concluded that even children with low disease activity may have some gait abnormalities. Inflammation of joints in the lower extremities may be associated with loss of muscle force, limited range of motion in the joints, as well as with asymmetric distribution of the center of gravity [14]. Abnormal motor development becomes more and more visible with age when more and more complex motor skills appear [27].

There are only few studies regarding a biomechanical analysis of gait in children with JIA [5], [11], [12], [20]. Some authors focused on the analysis of gait abnormalities resulting from disease activity [7], [11], [12]. Three-dimensional gait analysis (3DGA) was also used to assess abnormalities of a foot position and functions, and it was possible thanks to the use of the Oxford Foot Model protocol [18], [19]. Moreover, efficacy of treatment for the ankle joint with corticosteroid injections [9], [27] was assessed, and the effects of shockwave therapy on knee functions were studied [1]. In her work [9], Esbjörnsson assessed whether it was possible to use Gait Deviation Index (GDI) in the analysis of gait in children with JIA following corticosteroids injections into the ankle joint. Mayer et al. [20] used 3DGA in a 10-year-old girl in order to assess the efficacy of neuromuscular training. There are also reports regarding the efficacy of various types of rehabilitation programs that evaluated gait efficacy using 6-minute or 9-minute walking tests [16], [24][26] but an assessment of the gait quality was not considered. Currently, there are only results of one study regarding a gait assessment in relation to kinematic, spatiotemporal parameters and GDI in this group of patients available [9], and, moreover, there are no prospective studies assessing gait patterns and their changes following a complex treatment program. Therefore, this study aims to assess the gait pattern in children and adolescents with JIA compared to healthy individuals as well as to assess changes in the gait pattern in relation to kinematic, spatiotemporal parameters and GDI directly after the end of a rehabilitation program and nine months later.

\section{Materials and methods}

\subsection{Participants}

The study included children with JIA who were treated at Regional Centre for Rehabilitation and
Education for Children and Adolescents, Rzeszów, Poland. JIA was diagnosed according to the 2004 ILAR classification criteria [21]. Inclusion criteria for the study group were as follows: age between 6 and 18 years, confirmed diagnosis of JIA, disease remission, ability to walk 5 meters at least six times (independently or with elbow crutches), no intraarticular injections into affected joints within the preceding four weeks.

Study exclusion criteria were as follows: coexisting congenital or acquired (post-trauma) locomotor organ deficits not associated with JIA, past surgical treatment of lower extremities, coexisting neurological conditions or other problems potentially affecting the gait pattern. 50 subjects were in the study group after all criteria had been reviewed. The mean time since the diagnosis of the condition was $2.9 \pm 2.35$. The mean value of pain intensity based on the Visual Analogue Scale (VAS) (0-100) was 34.6. Pharmacotherapy included the following groups of medicines: disease-modifying anti-rheumatic drugs ( $80 \%$ of patients), steroidal anti-inflammatory drugs ( $26 \%$ of patients), biologicals (12\% of patients). Patients were allowed to take more than one type of medicines. The control group was added to the study in order to compare results. The control group included 35 healthy children without gait disorders, who attended primary, secondary schools in southeastern Poland. The control group was appropriately matched to the age (mean age $12.08 \pm 4.5$ years) and sex ( 25 girls, 10 boys) of the study group. Clinical characteristics of the study and control groups are presented in Table 1.

Oligoarthritis was observed the most frequently in $50 \%$ of children comprising the study group, and only one patient had systemic arthritis. When children with two the most common forms, such as polyarthritis and oligoarthritis were compared, it was concluded that there were no statistically significant differences between subjects regarding baseline characteristics. In Table 1, characteristics of the study group taking into account sub-types (polyarthritis and oligoarthritis) are presented. The difference between the groups was not statistically significant.

According to the Declaration of Helsinki all subjects and their legal guardians were informed about the purpose and course of the study and about their rights to withdraw from the study. All of them gave their written, informed and voluntary consent for participation in the study. The study protocol was approved by the local Bioethics Commission of the Medical Faculty of the University of Rzeszów $(17 / 02 / 2013)$. 
Table 1. Characteristics of the study group (subtypes in study group: oligoarthritis, polyarthritis) and control group

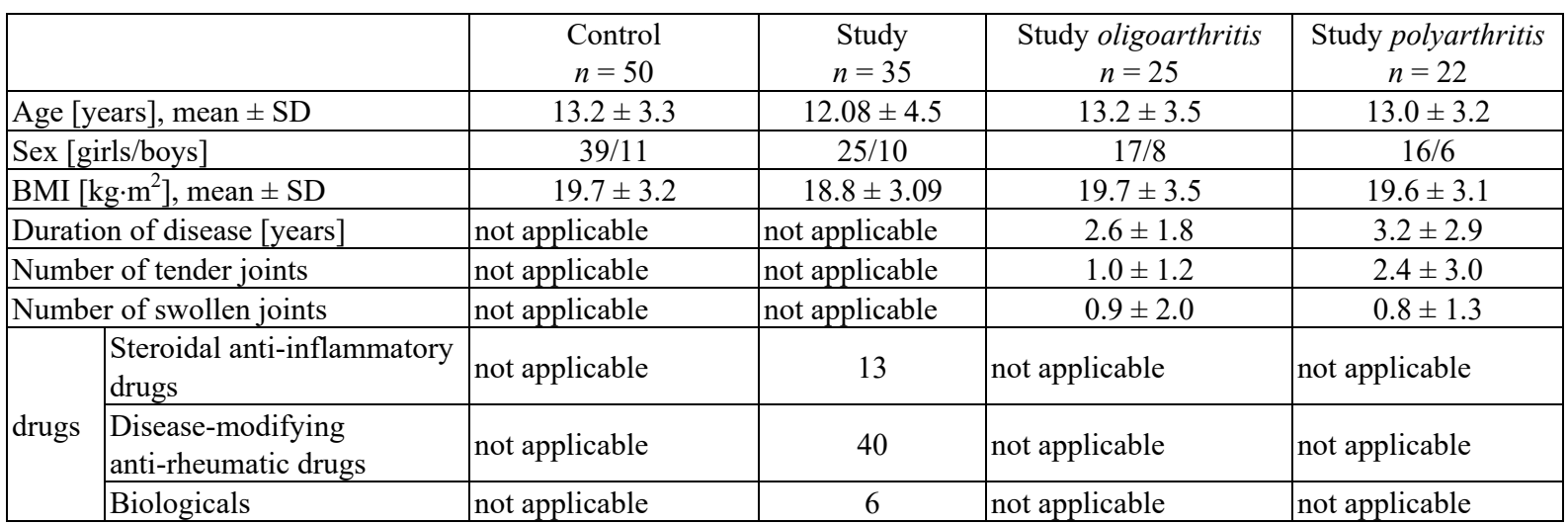

BMI - Body Mass Index, $n$ - number of subjects, SD - standard deviation.

\subsection{Procedure and measures}

Three assessments were scheduled in the study group: baseline tests at the beginning of rehabilitation at the center (Test 1), on the day when rehabilitation was completed at the center (Test 2) - rehabilitation duration was 4 weeks - and 9 months after test 2 (Test 3 ). There was one assessment in the control group.

Gait analysis was performed in the Biomechanics Laboratory at the University of Rzeszów, Department of Physiotherapy. The 3D analysis system applied was based on BTS Smart with six infrared cameras recording at $250 \mathrm{~Hz}$ (BTS SMART-DX 700, $250 \mathrm{~Hz}$; BTS Bioengineering, Milano, Italy) with software, in SMART Capture, Tracker and Analyzer (BTS Bioengineering, Milano, Italy). Passive reflective markers were attached with double-sided adhesive tape to 19 anatomical reference points, following the protocol proposed by Davis [8]. The markers were positioned at the sacrum, anterior superior iliac spine, greater trochanter, lateral femoral condyle, head of fibula, lateral malleolus, head of the 5th metatarsal and heel. Two tic bands with two markers were secured on the lateral aspect of both thighs and lower legs. The position of each marker was recorded 3-dimensionally at $250 \mathrm{~Hz}$. The system was calibrated before each test. After the subject of the study was assessed in the standing position, they were instructed on how to walk the specified distance of 5 metres. The subjects walked barefoot, at a comfortable speed. Consecutive trips over the specified pathway were recorded until 6-8 records with no artefacts were registered. The spatiotemporal data from gait analysis were then computed using BTS Smart software (Smart Tracker and Smart Analyzer). Data processing with Smart Tracker and Smart Analyzer software included defini- tion of gait events, i.e., initial contact and heel off for each leg; these were determined based on the signal from force platforms and on video recording.

\section{Spatiotemporal and kinematic parameters}

The following parameters were included in the analysis: 1) spatiotemporal: stance time [\%], swing time [\%], double stance time [\%], normalized step length, cadency [steps/min], normalized mean velocity, and step width [m]. With regard to step length and mean gait velocity adjustment was made in relation to limb length to allow for comparisons of children of various height (normalization of the step length was performed according to Hof [13] - normalized step length $=$ step length/limb length, while normalized gait velocity was calculated according to Bohannon [3] - normalized velocity $=$ gait velocity/limb length); 2) kinematic: pelvic tilt range of motion (ROM), pelvic tilt - initial contact (IC), pelvic obliquity - IC, maximum pelvic obliquity (Pelvic Obliquity Max), hip flexion/extension - IC (Hip Flex/Ext-IC), maximum hip extension-pre swing (Hip Ext-PSw Max), maximum hip flexion - mid swing (Hip Flex-MSw Max), hip flexion/extension ROM (Hip Flex/Ext ROM), knee flexion/extension-IC, maximum knee flexionmid swing (Knee Flex-MSw Max), maximum knee extension-terminal stance (Knee Ext-TSt Max), knee flexion/extension ROM (Knee Flex/Ext ROM), ankle dorsal flexion (Ankle Dors Flex), ankle plantar flexion (Ankle Plant Flex), ankle dorsal/plantar ROM (Ankle Dors/Plant ROM).

\section{GDI calculations}

The GDI was calculated based on kinematic data obtained from 3DGA. The GDI is developed using kinematic data from the pelvis, hip, knee, ankle and foot, as an average value of several gait cycles. In the 
sagittal plane, the GDI incorporates kinematics from the pelvis, hip, knee and ankle joint. In the frontal plane, the GDI incorporates kinematics from the pelvis and hip joint. In addition, the kinematics from pelvis, hip and foot progression in the transversal plane are included in the GDI. In total, the GDI includes 459 kinematic data points (nine angular kinematic variables were captured 51 times, i.e., every $2 \%$ of the gait cycle). These 459 data points are then compressed, and 15 parameters retrieved. These parameters are used to express an overall deviation of the subject's gait, compared to normal values of the 15 corresponding parameters in the control group [23].

A healthy reference group was necessary to calculate GDI. A separate numeric value is given for the right and left limb. In order to obtain GDI values, we used an Excel spreadsheet that was developed by Schwartz and Rozumalski [23] was used. According to these authors, the degree of gait pathology can be determined by calculating the distance between the subject's gait vector and a mean vector obtained from a healthy reference group (without gait disorders). GDI $\geq 100$ represents gait which is at least as close to the healthy reference group mean as that of a randomly selected healthy individual. In other words, a result of 100 or more indicates a normal gait pattern, whereas each reduction by 10 points indicates one standard deviation (SD) from the normal gait [23].

\subsection{Statistical analysis}

Distribution of values of performance measures in the study group (in individual tests) was compared to results obtained in the control group. The MannWhitney $U$-test was used to assess whether differences between groups were significant. The Wilcoxon test was used to assess significance of therapeutic effects. The findings also comprise results of the analyses which tested the significance of the correlation coefficient $(r)$. The level of significance was set at $p \leq 0.05$. The level of statistical significance was corrected using Bonferroni adjustment for multiple comparisons (threefold evaluation). Statistical significance was assumed at $p<0.017$. The choice of tests applied to examine the variables depended on whether or not the basic assumptions were fulfilled regarding the agreement between distributions of the relevant variables and normal distribution. Parameters of the characteristics distribution were assessed with the use of Shapiro-Wilk $U$-test, which indicated abnormal distribution.
The study and the control group were also divided into four groups in relation to the normalized mean gait velocity. The grouping was performed separately for the study and control group, concerning the normalized mean gait velocity quartiles values as a cutoff points. Then the comparison of normalized mean gait velocity between the study and control group, in the first, second and third test was performed, within the same range of normalized mean gait velocity (quartiles). Statistical analyses were conducted with the use of Statistica 10.0 program (StatSoft, Poland).

\section{Results}

The flow of the subjects through the study is shown in Fig. 1. Test 1 included 72 subjects meeting inclusion criteria, Test $2-67$ subjects, and Test $3-50$ subjects. 22 subjects were lost to follow-up due to the following reasons: 5 subjects - completed rehabilitation at the center earlier than after 4 weeks, 10 subjects - did not come for the test after 9 months, 7 subjects - did not consent for the test after 9 months.

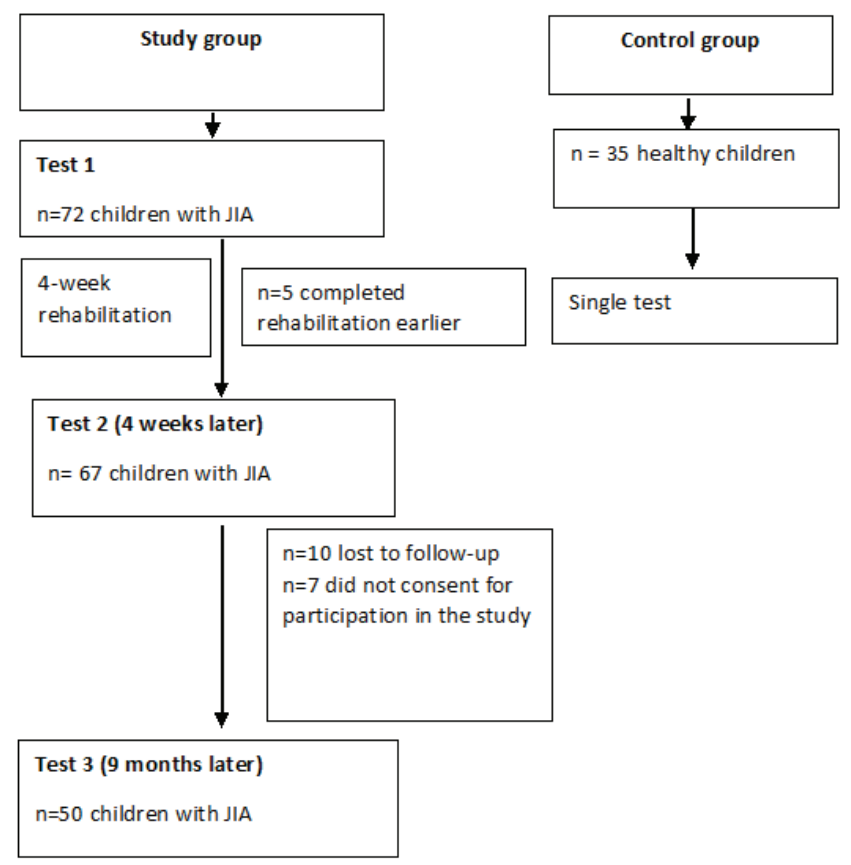

Fig. 1. The flow of the subjects through the study

At the beginning, the performance status before treatment was compared taking into account two the most common types of JIA (oligoarthritis and polyarthritis). When spatiotemporal, kinematic parameters and GDI were analyzed in the baseline test before treatment, there were no significant differences be- 
tween variables studied except for normalized step length for the left extremity. In patients with oligoarthritis, it was longer only for the left lower extremity $p=0.0279$. Therefore, a decision was made to analyze gait in the whole group of 50 children without stratification based on JIA in further analyses.

\subsection{Comparison of results in the study group and control group in Tests 1,2 and 3}

Differences between groups were assessed with regard to significance in order to answer the question of whether there are any differences regarding parameters assessed in a group of children with JIA compared to a group of healthy children.

\section{Spatiotemporal parameters}

With regard to the majority of spatiotemporal parameters, differences between their distribution in the study group in Test 1 compared to the control group were highly statistically significant $(p<0.001)$ for both right and left lower extremity (Table 2). Stance time and swing time were within generally accepted reference range of $60-40 \%$; however, stance time was longer compared to the control group. A similar situation was observed for double stance time. Children with JIA walked more slowly (normalized mean velocity 1.4 ) and with smaller cadence (110.8 steps/minute) - Table 2. Individual spatiotemporal parameters in two subsequent tests were also compared to the control group. Differences in relation to the control group were less visible $(p<0.01$ or even only $p<0.05$ for normalized mean velocity). Although there was improvement visible in parameters studied, there were still differences in Tests 2 and 3, namely after 9 months, compared to the control group (Table 2).

Normalized mean gait velocity in the study and control group, in the first, second and third test, separately for each of the 4 subgroups (regarding gait velocity) was the subject of further statistical analysis. In the first test (Test 1), in all four groups (Q1, Q2, Q3, Q4), the normalized mean gait velocity was significantly lower in the study group than in the control group. In the second test (Test 2), significantly lower gait velocity were observed in the Q2 group ( $p=0.0011)$ and Q4 group ( $p=0.0111)$ in the study group. In the third test (Test 3 ), significantly lower gait velocity were only observed in the Q2 group ( $p=0.0071$ ) of the study group, compared to the healthy subjects (Table 3).

Table 2. List of spatiotemporal parameters in the study group compared to the control group in Tests 1 , Test 2 and Test 3

\begin{tabular}{|l|c|c|c|c|c|c|}
\hline \multirow{2}{*}{$\begin{array}{c}\text { Spatiotemporal } \\
\text { parameters }\end{array}$} & \multicolumn{2}{|c|}{ Test 1 } & \multicolumn{2}{c|}{ Test 2 } & \multicolumn{2}{c|}{ Test 3 } \\
\cline { 2 - 7 } & Study group & Control group & Study group & Control group & Study group & Control group \\
\hline $\begin{array}{l}\text { Stance phase rl [\% gait cycle] } \\
\text { mean } \pm \text { SD }\end{array}$ & $60.5 \pm 1.3$ & $58.9 \pm 1.6^{(\mathrm{b})}$ & $60.3 \pm 1.3$ & $58.9 \pm 1.6^{(\mathrm{b})}$ & $60.4 \pm 1.4$ & $58.9 \pm 1.6^{(\mathrm{b})}$ \\
\hline $\begin{array}{l}\text { Swing phase rl [\% gait cycle] } \\
\text { mean } \pm \text { SD }\end{array}$ & $39.5 \pm 1.3$ & $41.1 \pm 1.6^{(\mathrm{b})}$ & $39.6 \pm 1.3$ & $41.1 \pm 1.6^{(\mathrm{b})}$ & $39.6 \pm 1.5$ & $41.1 \pm 1.6^{(\mathrm{b})}$ \\
\hline $\begin{array}{l}\text { Double stance rl [\% gait cycle] } \\
\text { mean } \pm \text { SD }\end{array}$ & $10.4 \pm 1.4$ & $9.1 \pm 1.8^{(\mathrm{b})}$ & $10.3 \pm 1.4$ & $9.1 \pm 1.8\left(^{\mathrm{b}}\right)$ & $10.5 \pm 1.6$ & $9.1 \pm 1.8^{(\mathrm{b})}$ \\
\hline $\begin{array}{l}\text { Normalized step length rl [m] } \\
\text { mean } \pm \text { SD }\end{array}$ & $0.64 \pm 0.09$ & $0.71 \pm 0.08^{(\mathrm{b})}$ & $0.66 \pm 0.09$ & $0.71 \pm 0.08^{(*)}$ & $0.65 \pm 0.09$ & $0.71 \pm 0.08^{(\mathrm{b})}$ \\
\hline $\begin{array}{l}\text { Stance phase 11 [\% gait cycle] } \\
\text { mean } \pm \text { SD }\end{array}$ & $60.6 \pm 2.3$ & $58.8 \pm 1.6^{(\mathrm{b})}$ & $60.6 \pm 2.2$ & $58.8 \pm 1.6^{(\mathrm{b})}$ & $60.3 \pm 1.7$ & $58.8 \pm 1.6^{(\mathrm{b})}$ \\
\hline $\begin{array}{l}\text { Swing phase 11 [\% gait cycle] } \\
\text { mean } \pm \text { SD }\end{array}$ & $39.4 \pm 2.3$ & $41.2 \pm 1.6^{(\mathrm{b})}$ & $39.4 \pm 2.3$ & $41.2 \pm 1.6^{(\mathrm{b})}$ & $39.7 \pm 1.7$ & $41.2 \pm 1.6^{(\mathrm{b})}$ \\
\hline $\begin{array}{l}\text { Double stance ll [\% gait cycle] } \\
\text { mean } \pm \text { SD }\end{array}$ & $10.6 \pm 1.5$ & $8.7 \pm 1.6^{(\mathrm{b})}$ & $10.4 \pm 1.7$ & $8.7 \pm 1.6^{(\mathrm{b})}$ & $10.4 \pm 1.5$ & $8.7 \pm 1.6^{(\mathrm{b})}$ \\
\hline $\begin{array}{l}\text { Normalized step length 11 [m] } \\
\text { mean } \pm \text { SD }\end{array}$ & $0.64 \pm 0.08$ & $0.71 \pm 0.07^{(\mathrm{b})}$ & $0.67 \pm 0.09$ & $0.71 \pm 0.07^{(*)}$ & $0.66 \pm 0.07$ & $0.71 \pm 0.07^{(\mathrm{b})}$ \\
\hline Cadency [steps/min] mean \pm SD & $110.8 \pm 10.7$ & $122 \pm 14^{(\mathrm{b})}$ & $113 \pm 10.8$ & $122 \pm 14^{(\mathrm{b})}$ & $113.1 \pm 12.1$ & $122.0 \pm 14.0^{(\mathrm{b})}$ \\
\hline $\begin{array}{l}\text { Normalized mean velocity [m/s] } \\
\text { mean } \pm \text { SD }\end{array}$ & $1.4 \pm 0.28$ & $1.58 \pm 0.31^{(\mathrm{b})}$ & $1.47 \pm 0.27$ & $1.58 \pm 0.31$ & $1.48 \pm 0.30$ & $1.58 \pm 0.31$ \\
\hline Step width [m] & $0.15 \pm 0.03$ & $0.15 \pm 0.03$ & $0.14 \pm 0.03$ & $0.15 \pm 0.03$ & $0.15 \pm 0.03$ & $0.15 \pm 0.03$ \\
\hline
\end{tabular}

$\mathrm{rl}$ - right limb, 11 - left limb, SD - standard deviation; statistically significant changes $(p<0.05)$ are marked with asterisk ${ }^{(*)}$; significant differences between the groups after Bonferroni correction $(p<0.017)$ are marked with ${ }^{(b)}$. 
Table 3. Comparisons of the interquartile range of gait velocity between the study and control group, in Tests 1, 2 and 3

\begin{tabular}{|c|c|c|}
\hline \multirow{3}{*}{$\begin{array}{l}\text { Interquartile range } \\
\text { of gait velocity }\end{array}$} & \multicolumn{2}{|c|}{ Normalized mean gait velocity } \\
\hline & \multicolumn{2}{|c|}{ Test 1} \\
\hline & Study group (mean $\pm \mathrm{SD})$ & Control group (mean $\pm \mathrm{SD})$ \\
\hline $\mathrm{Q} 1[\mathrm{~m} / \mathrm{s}]$ & $1.07 \pm 0.20$ & $1.24 \pm 0.15\left(^{b}\right)$ \\
\hline $\mathrm{Q} 2[\mathrm{~m} / \mathrm{s}]$ & $1.31 \pm 0.04$ & $1.46 \pm 0.03\left(^{b}\right)$ \\
\hline $\mathrm{Q} 3[\mathrm{~m} / \mathrm{s}]$ & $1.48 \pm 0.08$ & $1.60 \pm 0.08\left(^{b}\right)$ \\
\hline \multirow[t]{3}{*}{$\mathrm{Q} 4[\mathrm{~m} / \mathrm{s}]$} & $1.74 \pm 0.16$ & $2.00 \pm 0.19\left(^{b}\right)$ \\
\hline & \multicolumn{2}{|c|}{ Test 2} \\
\hline & Study group (mean $\pm \mathrm{SD})$ & Control group $($ mean $\pm \mathrm{SD})$ \\
\hline $\mathrm{Q} 1[\mathrm{~m} / \mathrm{s}]$ & $1.12 \pm 0.22$ & $1.24 \pm 0.15$ \\
\hline $\mathrm{Q} 2[\mathrm{~m} / \mathrm{s}]$ & $1.39 \pm 0.04$ & $1.46 \pm 0.03\left(^{b}\right)$ \\
\hline $\mathrm{Q} 3[\mathrm{~m} / \mathrm{s}]$ & $1.55 \pm 0.06$ & $1.60 \pm 0.08$ \\
\hline \multirow[t]{3}{*}{$\mathrm{Q} 4[\mathrm{~m} / \mathrm{s}]$} & $1.79 \pm 0.11$ & $2.00 \pm 0.19\left(^{b}\right)$ \\
\hline & \multicolumn{2}{|c|}{ Test 3} \\
\hline & Study group $($ mean $\pm \mathrm{SD})$ & Control group $($ mean $\pm \mathrm{SD})$ \\
\hline $\mathrm{Q} 1[\mathrm{~m} / \mathrm{s}]$ & $1.14 \pm 0.17$ & $1.24 \pm 0.15$ \\
\hline $\mathrm{Q} 2[\mathrm{~m} / \mathrm{s}]$ & $1.39 \pm 0.05$ & $1.46 \pm 0.03\left({ }^{b}\right)$ \\
\hline $\mathrm{Q} 3[\mathrm{~m} / \mathrm{s}]$ & $1.55 \pm 0.05$ & $1.60 \pm 0.08$ \\
\hline $\mathrm{Q} 4[\mathrm{~m} / \mathrm{s}]$ & $1.85 \pm 0.22$ & $2.00 \pm 0.19$ \\
\hline
\end{tabular}

Test 1 - baseline tests at the beginning of rehabilitation, Test 2 - on the day when rehabilitation was completed, Test 3 - follow up 9 months after test $2, \mathrm{SD}$ - standard deviation, statistically significant changes $(p<0.05)$ are marked with asterisk $\left.{ }^{*}\right)$, significant differences between the groups after Bonferroni correction $(p<0.017)$ are marked with ${ }^{(b)}$.

\section{Kinematic parameters}

In the period before treatment, there was an extremely statistically significant difference between groups for the majority of kinematic parameters (Tables 5, 6). In the study group, Pelvic Tilt-IC (right $p=0.0000$, left $p=0.0002$ ) and Hip Flex/Ext-IC were increased (left limb $p=0.0078$ ). On the other
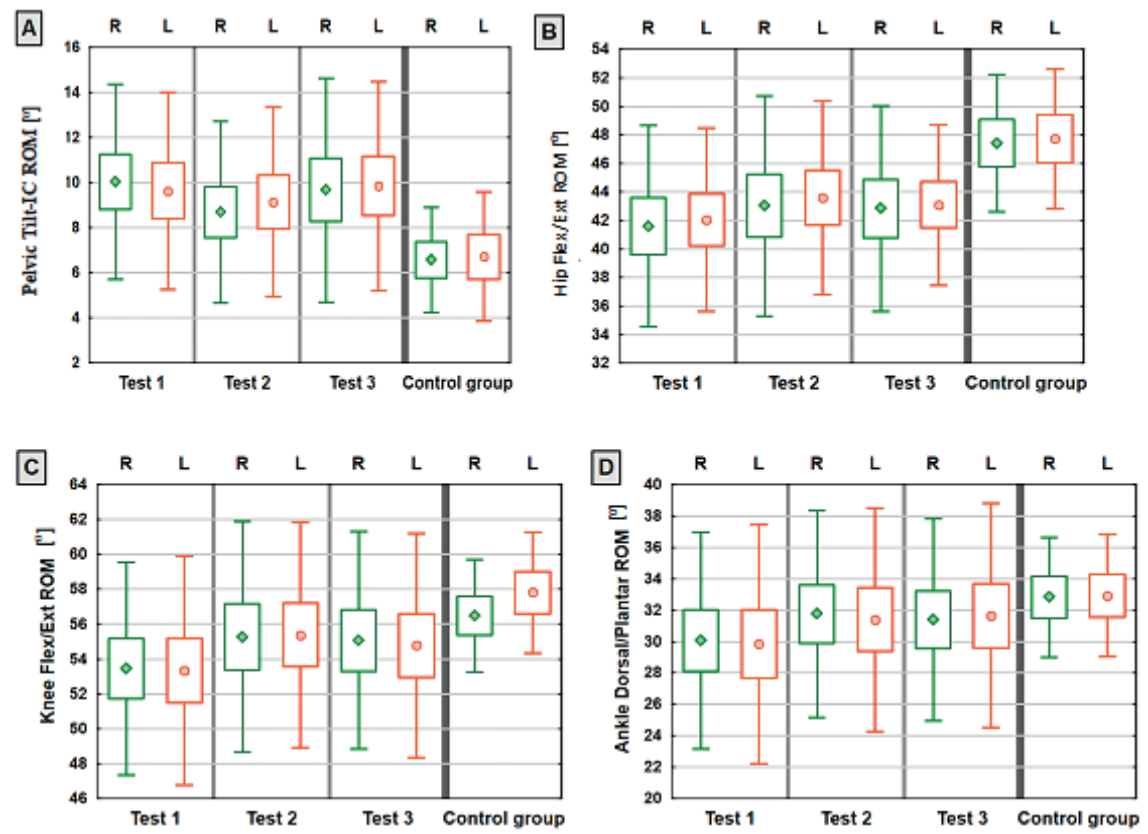

Fig. 2. Comparisons of the pelvic tilt IC, Hip Flex/Ext ROM, Knee Flex/Ext ROM, Ankle Dors/Plant ROM for the right and left size in the two groups and the three tests (mean, $95 \%$ confidence interval): $\mathrm{R}$ - right limb, $\mathrm{L}$ - left limb, ROM - range of motion, IC - initial contact, Flex - flection, Ext - extension, Dors - dorsal, Plant - plantar 
Table 4. List of kinematic parameters in the study group compared to the control group in Test 1, Test 2 and Test 3

\begin{tabular}{|c|c|c|c|c|c|c|c|}
\hline \multirow{3}{*}{\multicolumn{2}{|c|}{ Kinematic parameters }} & \multicolumn{2}{|c|}{ Test 1} & \multicolumn{2}{|c|}{ Test 2} & \multicolumn{2}{|c|}{ Test 3} \\
\hline & & Study group & Control group & Study group & Control group & Study group & Control group \\
\hline & & mean $\pm \mathrm{SD}$ & mean $\pm \mathrm{SD}$ & mean $\pm \mathrm{SD}$ & mean $\pm \mathrm{SD}$ & mean $\pm \mathrm{SD}$ & mean $\pm \mathrm{SD}$ \\
\hline \multirow{6}{*}{ 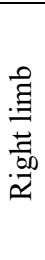 } & Pelvic Tilt-IC ROM $\left[^{\circ}\right]$ & $10.03 \pm 4.33$ & $6.58 \pm 2.33^{(\mathrm{b})}$ & $8.69 \pm 4.03$ & $6.58 \pm 2.33^{(b)}$ & $9.67 \pm 4.97$ & $6.58 \pm 2.33^{(\mathrm{b})}$ \\
\hline & Hip Flex/Ext-IC ROM [ $\left.{ }^{\circ}\right]$ & $37.8 \pm 7.0$ & $35.1 \pm 3.9^{(*)}$ & $37.8 \pm 6.0$ & $35.1 \pm 3.9^{(b)}$ & $38.4 \pm 6.9$ & $35.1 \pm 3.9^{(\mathrm{b})}$ \\
\hline & Hip Ext-PSw Max ROM [ $\left.{ }^{\circ}\right]$ & $2.01 \pm 7.60$ & $10.66 \pm 3.73^{(\mathrm{b})}$ & $3.57 \pm 7.17$ & $10.66 \pm 3.73^{(\mathrm{b})}$ & $3.61 \pm 7.27$ & $10.66 \pm 3.73^{(b)}$ \\
\hline & Hip Flex/Ext ROM [] & $41.6 \pm 7.0$ & $47.4 \pm 4.8^{(\mathrm{b})}$ & $43.0 \pm 7.7$ & $47.4 \pm 4.8^{(\mathrm{b})}$ & $42.8 \pm 7.2$ & $47.4 \pm 4.8^{(\mathrm{b})}$ \\
\hline & Knee Flex/Ext-IC ROM $\left[^{\circ}\right]$ & $14.7 \pm 5.6$ & $10.1 \pm 2.2^{(\mathrm{b})}$ & $15.8 \pm 5.6$ & $10.1 \pm 2.2^{(b)}$ & $16.3 \pm 5.1$ & $10.1 \pm 2.2^{(\mathrm{b})}$ \\
\hline & Knee Flex-MSw Max ROM $\left[^{\circ}\right]$ & $63.9 \pm 8.0$ & $61.5 \pm 3.1^{(\mathrm{b})}$ & $66.0 \pm 8.3$ & $61.5 \pm 3.1^{(\mathrm{b})}$ & $66.5 \pm 7.4$ & $61.5 \pm 3.1^{(\mathrm{b})}$ \\
\hline \multirow{9}{*}{ 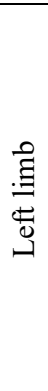 } & Pelvic Tilt-IC ROM $\left[{ }^{\circ}\right]$ & $9.63 \pm 4.37$ & $6.71 \pm 2.86^{(\mathrm{b})}$ & $9.14 \pm 4.21$ & $6.71 \pm 2.86^{(\mathrm{b})}$ & $9.85 \pm 4.64$ & $6.71 \pm 2.86^{(\mathrm{b})}$ \\
\hline & Hip Flex/Ext-IC ROM $\left[^{\circ}{ }^{\circ}\right.$ & $38.2 \pm 6.4$ & $35.2 \pm 4.6^{(\mathrm{b})}$ & $38.5 \pm 6.4$ & $35.2 \pm 4.6^{(\mathrm{b})}$ & $39.0 \pm 7.0$ & $35.2 \pm 4.6^{(\mathrm{b})}$ \\
\hline & Hip Ext-PSw Max ROM [ $\left.{ }^{\circ}\right]$ & $1.64 \pm 7.01$ & $11.23 \pm 3.51^{(\mathrm{b})}$ & $3.25 \pm 7.13$ & $11.23 \pm 3.51^{(\mathrm{b})}$ & $2.56 \pm 8.00$ & $11.23 \pm 3.51^{(\mathrm{b})}$ \\
\hline & Hip Flex/Ext ROM [] & $42.0 \pm 6.4$ & $47.7 \pm 4.9^{(\mathrm{b})}$ & $43.6 \pm 6.8$ & $47.7 \pm 4.9^{(\mathrm{b})}$ & $43.1 \pm 5.6$ & $47.7 \pm 4.9^{(\mathrm{b})}$ \\
\hline & Knee Flex/Ext-IC ROM $\left[^{\circ}\right]$ & $14.9 \pm 4.8$ & $8.9 \pm 2.4^{(b)}$ & $16.5 \pm 5.5$ & $8.9 \pm 2.4^{(b)}$ & $17.1 \pm 6.1$ & $8.9 \pm 2.4^{(b)}$ \\
\hline & Knee Flex-MSw Max ROM $\left[^{\circ}\right]$ & $64.9 \pm 5.9$ & $61.7 \pm 3.1^{(\mathrm{b})}$ & $67.1 \pm 6.8$ & $61.7 \pm 3.1^{(\mathrm{b})}$ & $66.9 \pm 7.8$ & $61.7 \pm 3.1^{\text {(b) }}$ \\
\hline & Knee Flex/Ext-TSt Max ROM [ $\left.{ }^{\circ}\right]$ & $13.3 \pm 6.0$ & $4.3 \pm 2.4^{(b)}$ & $12.8 \pm 5.6$ & $4.3 \pm 2.4^{(\mathrm{b})}$ & $12.9 \pm 7.1$ & $4.3 \pm 2.4^{(b)}$ \\
\hline & Knee Flex/Ext ROM $\left[{ }^{\circ}\right]$ & $53.3 \pm 6.6$ & $57.8 \pm 3.5^{(b)}$ & $55.4 \pm 6.4$ & $57.8 \pm 3.5$ & $54.8 \pm 6.4$ & $57.8 \pm 3.5^{(\mathrm{b})}$ \\
\hline & Ankle Dors/Plant ROM $\left[{ }^{\circ}\right]$ & $29.8 \quad 7.6$ & $32.93 .9^{(\mathrm{b})}$ & $31.4 \quad 7.1$ & $32.2 \quad 3.5$ & $31.7 \quad 7.1$ & $31.9 \quad 3.7$ \\
\hline
\end{tabular}

ROM - range of motion, IC - initial contact, Flex - flection, Ext - extension, PSw - pre swing, MSw - mid stance, Dors - dorsal, Plant - plantar, SD - standard deviation, statistically significant changes $(p<0.05)$ are marked with asterisk ${ }^{(*)}$, significant differences between the groups after Bonferroni correction $(p<0.017)$ are marked with ${ }^{(b)}$.

hand, Hip Ext-PSw Max was significantly reduced (mean for the right limb $2.01^{\circ}$, left limb $1.64^{\circ}$ ) $-p=0.0000$. In the whole gait cycle, Hip Flex/Ext ROM was smaller for both right and left limb, with significant statistical significance $(p=0.0000)$. Additionally, there was a significant difference regarding kinematic parameters of the knee joint. In children in the study group, the Knee Flex-MSw Max was increased (right limb $p=0.0009$; left limb $p=0.0008$ ) and Knee Flex/Ext ROM was reduced in the whole gait cycle (right limb $p=0.0054$; left $\operatorname{limb} p=0.0004$ ). With regard to the ankle, reduced Ankle Dors/Plant ROM on the left $(p=0.0128)$ were observed. The selected, representative figures (statistically significant results) of the kinematic variables (pelvis, hip, knee and ankle) for the two groups and the three comparisons were shown in Fig. 2. Moreover, a comparison of selected kinematic parameters in Tests 2 and 3 in relation to the control group was also performed to determine whether values of these angular parameters were closer to normal values after treatment. It was observed that in Tests 2 and 3 there were significant differences between the study and control groups for the majority of kinematic parameters; however, the scale of these differences was slightly smaller for some of these parameters when compared to values before rehabilitation ( $\mathrm{p}$ values (calculated probability) increased) - Table 5 and Table 6 . Table 4 presents statistically significant results.

\section{The Gait Deviation Index}

Lower GDI scores were reported for the right limb (Test 1: mean 94.9, SD 8.4; Test 2: mean 96.9, SD 9.2; Test 3: mean 94.5, SD 7.0), and for the left limb (Test 1: mean 96.4, SD 10.6; Test 2: mean 96.1, SD 10.6; Test 3: mean 93.2, SD 9.7) in the study group compared to healthy subjects (mean 100.00, SD 10.0). A comparison of GDI between the study and control groups in subsequent tests indicated its significantly lower value for both limbs in Test 3 (GDI left limb

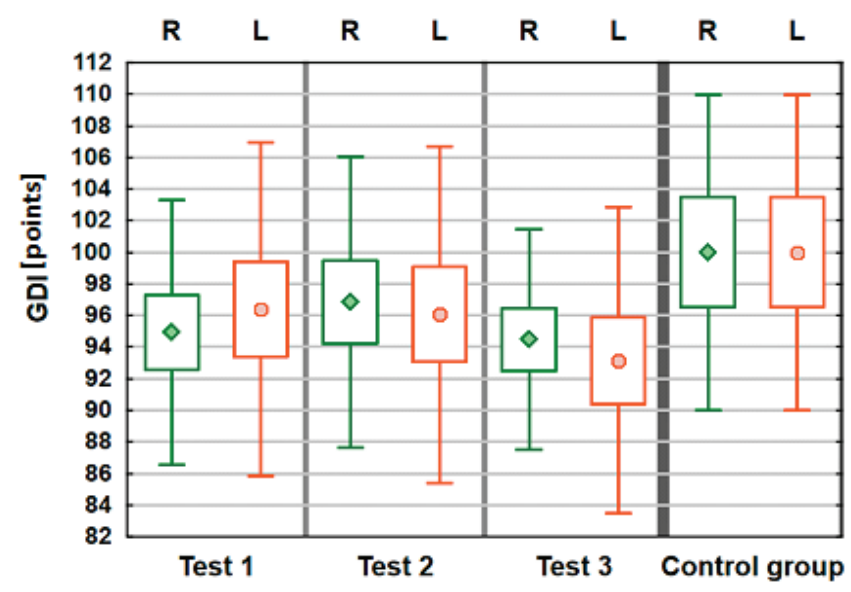

Fig. 3. Comparisons of the GDI values for the right/left limb in the study and control group in Tests 1,2 and 3

(mean, 95\% confidence interval): GDI - Gait Deviation Index, $\mathrm{R}$ - right limb, $\mathrm{L}$ - left limb 
$p=0.0036$; GDI right $\operatorname{limb} p=0.0095$ ). It was possible to observe that the gait pattern in children with JIA was comparable to that of healthy children immediately after the end of treatment. However, it worsened after 9 months since rehabilitation at the ward (Fig. 3).

\subsection{Comparison of results in the study group in Test 1 , Test 2 and Test 3}

\section{Spatiotemporal parameters}

The normalized step length for the right and left limb statistically significantly increased immediately after treatment ( $p=0.0078, p=0.0031$, respectively); however, improvement was not maintained during further tests. Cadence increased after treatment ( $p=0.0016)$, and it was a permanent effect. A difference between Test 1, Test 2 and Test 3 was on average 2.2-2.3 steps per minute. Normalized mean gait velocity increased as a result of treatment. This effect was statistically significant ( $p=0.0007)$. Within 9 subsequent months its range changed, but a visible tendency for worsening or improvement was not visible. Consequently, results after 9 months were significantly better compared to the ones before rehabilitation $(p=0.0062)$. In Table 5, statistically significant results are presented.

\section{Kinematic parameters}

In Table 6, changes in values of kinematic parameters between subsequent tests are shown. ROM in the sagittal plane in the hip joints, knee joints and ankle

Table 5. List of spatiotemporal parameters in the study group in subsequent tests

\begin{tabular}{|c|c|c|c|}
\hline \multirow[t]{2}{*}{ Spatiotemporal parameters } & \multicolumn{3}{|c|}{$\begin{array}{l}\text { Mean change between tests } \\
\text { (with } p \text {-value indicating statistical significance using the Wilcoxon test) }\end{array}$} \\
\hline & Test 1 vs. Test 2 & Test 2 vs. Test 3 & Test 1 vs. test 3 \\
\hline Stance phase rl [\% of gait cycle] & -0.2 & 0.0 & -0.1 \\
\hline Swing phase rl [\% of gait cycle] & 0.1 & -0.1 & 0.1 \\
\hline Double stance rl [\% of gait cycle] & -0.1 & 0.1 & 0.1 \\
\hline Normalized step length rl [m] & $0.02\left(^{b}\right)$ & -0.01 & 0.01 \\
\hline Stance phase 11 [\% of gait cycle] & 0.0 & -0.3 & -0.2 \\
\hline Swing pfase 11 [\% of gait cycle] & 0.0 & 0.4 & 0.3 \\
\hline Double stance 11 [\% of gait cycle] & -0.2 & 0.0 & -0.2 \\
\hline Normalized step length $11[\mathrm{~m}]$ & $0.02\left(^{b}\right)$ & -0.01 & 0.02 \\
\hline Cadency [steps/min] & $2.2\left(^{b}\right)$ & 0.1 & $2.3(*)$ \\
\hline Normalized mean velocity $[\mathrm{m} / \mathrm{s}]$ & $0.06\left(^{b}\right)$ & 0.01 & $0.08\left(^{b}\right)$ \\
\hline Step width [m] & 0.00 & 0.01 & $0.00(0.2945)$ \\
\hline
\end{tabular}

$\mathrm{rl}$ - right limb, 11 - left limb, SD - standard deviation, statistically significant changes $(p<0.05)$ are marked with $\operatorname{asterisk}^{(*)}$, significant differences between the groups after Bonferroni correction $(p<0.017)$ are marked with $\left(^{b}\right)$.

Table 6. List of kinematic parameters in the study group in subsequent tests

\begin{tabular}{|c|c|c|c|}
\hline \multirow{2}{*}{ Kinematic parameters } & \multicolumn{3}{|c|}{$\begin{array}{l}\text { Mean change between tests } \\
\text { (with } p \text {-value indicating statistical significance using the Wilcoxon test) }\end{array}$} \\
\hline & Test 1 vs. Test 2 & Test 2 vs. Test 3 & Test 1 vs. Test 3 \\
\hline \multicolumn{4}{|c|}{ Right limb } \\
\hline Hip Flex/Ext ROM [ $\left.{ }^{\circ}\right]$ & $1.4\left(^{b}\right)$ & -0.2 & $1.2\left(^{b}\right)$ \\
\hline Knee Flex-MSw Max ROM [] & $2.1\left(^{b}\right)$ & 0.5 & $2.6\left(^{b}\right)$ \\
\hline Knee Flex/Ext ROM $\left[^{\circ}\right]$ & $1.8\left(^{b}\right)$ & -0.2 & $1.6^{(*)}$ \\
\hline Ankle Dors/Plant ROM $\left[^{\circ}\right]$ & $1.7\left(^{b}\right)$ & -0.4 & 1.3 \\
\hline \multicolumn{4}{|c|}{ Left Limb } \\
\hline Hip Flex/Ext ROM [ $\left.{ }^{\circ}\right]$ & $1.5\left(^{b}\right)$ & -0.5 & $1.1^{(*)}$ \\
\hline Knee Flex-MSw Max ROM $\left[^{\circ}\right]$ & $2.2\left(^{b}\right)$ & -0.2 & $2.0\left(^{b}\right)$ \\
\hline Knee Flex/Ext ROM $\left[^{\circ}\right]$ & $2.1\left(^{b}\right)$ & -0.6 & $1.4^{(*)}$ \\
\hline Ankle Dors Flex ROM $\left[{ }^{\circ}\right]$ & 0.5 & 0.8 & $1.3\left({ }^{b}\right)$ \\
\hline
\end{tabular}

ROM - range of motion, IC - initial contact, Flex - flection, Ext - extension, PSw - pre swing, MSw - mid stance, Dors - dorsal, Plant - plantar, SD - standard deviation, statistically significant changes $(p<0.05)$ are marked with asterisk $^{(*)}$, significant differences between the groups after Bonferroni correction $(p<0.017)$ are marked with ${ }^{(b)}$. 
joints increased significantly between Test 1 , Test 2 and Test 3. In Table 6, statistically significant results are presented.

\section{$G D I$}

The value of GDI for the right and left limb did not change statistically significantly between tests (Test 1 vs. Test 2: $p=0.1463$; Test 2 vs. Test $3: p=0.1812$; Test 1 vs. Test $3: p=0.7981)$. Individual changes observed in individual patients were sometimes quite significant, both positively and negatively, but averaged results of treatment were close to 0 .

\section{Discussion}

The study was designed to assess gait patterns in children with JIA, compared to gait patterns in healthy peers, and to perform prospective analysis of changes in gait pattern during nine months following a rehabilitation program. The current article is the first one to report the related evidence and present detailed description of the gait pattern in a large population of children with JIA.

The present findings related to most of the spatiotemporal and kinematic parameters in the study group and the controls showed that the differences in their distributions were highly statistically significant. In Test 3 , carried out nine months after the end of rehabilitation, the gait parameters which had differed between the groups in Test 1 and Test 2 still showed a statistically significant difference. It was also shown that the range of motion of the ankle was significantly lower in the study group only in Test 1 . In the subsequent assessments, this difference was not statistically significant. This may have been associated with the intensity of medical symptoms (pain, and swelling in the joints) which were observed in the patients prior to the rehabilitation. Lower intensity of pain following rehabilitation may have significantly contributed to an improvement in gait pattern. After nine months, the patients did not report pain, however their gait pattern still differed from that observed in the controls. It is possible that the persisting differences, including the greater range of motion of the hip and knee during initial contact and mid-stance phase in the study group, reflect a compensatory pain-induced gait pattern, or pattern caused by articular lesions. Nevertheless, this is important information suggesting objectives for further therapy and rehabilitation. Differences in gait patterns between children with JIA and healthy controls have also been reported by other researchers [5]-[7]. Lechner et al. [5] concluded that children with JIA presented lower walking speed, their step was shorter, but their step width was comparable to that of healthy children. Contrary to the current study, these researchers did not observe differences in the percentage division of the gait cycle, but they observed lower step frequency. With regard to kinematic parameters, the authors observed similar disturbances: increased pelvic tilt and hip flexion during the whole gait cycle, limited hip extension and limited plantar flexion in the ankle. Hartman et al. [6] also reported significant differences regarding all spatiotemporal and kinematic parameters assessed in their work. Likewise, Brostrom et al. [7] demonstrated that patients with JIA walked more slowly compared to their healthy peers.

In the current study, adjustment of parameters in relation to the length of the limb was conducted both for mean gait velocity and for step length and, therefore, it was possible to assess these parameters more reliably. The need for adjustment was especially visible in the case of step length in Test 1 where there were no differences for either the right or the left limb, compared to the control group. However, after baseline parameters were adjusted, the differences became visible. The current findings regarding spatiotemporal parameters are not consistent with reports by Merker et al. [14] which mainly aimed to assess the relationship between a planovalgus foot and gait function in children with JIA. They did not observe any significant differences with regard to spatiotemporal parameters in relation to the control group, although the value of this assessment is reduced by the small size of the study group (11 subjects with JIA). As regards the kinematic parameters, researchers did not demonstrate limited plantar flexion in relation to the control group, but they observed a deficit of dorsal flexion.

The current findings showed lower values of GDI in the study group compared to the healthy subjects. In Test 2, there were no statistically significant differences which were expected, such as an increase in the study variable, despite the fact that such improvement was observed in the specific kinematic parameters. It was possible to observe that the gait pattern in children with JIA was comparable to that of healthy children immediately after the end of the therapy. However, it deteriorated nine months after the rehabilitation at the ward. Based on the literature review, it can be concluded that only Esbjornsson et al. [16] used GDI to assess gait in this group of patients. The authors calculated the total GDI for the right and left lower limb at the same time. They did not observe differences in relation to the control group [16]. 
A comparison of the results related to the spatiotemporal parameters in the study group observed in Tests 1, 2 and 3, shows that the normalized step length significantly increased immediately after the treatment, however, this improvement was not retained during the subsequent tests. On the other hand, cadence increased after the treatment and it was a lasting effect. Normalized mean gait velocity increased as a result of the therapy. During the following months, its range changed, but a visible tendency for deterioration or improvement was not observed. Consequently, the results after nine months were significantly better compared to the scores before the rehabilitation. Analysis of the results identified in the study group in Tests 1, 2 and 3, regarding the kinematic parameters, shows that ranges of motion in the sagittal plane in the hip, knee and ankle joints increased significantly between Test 1 and 2, as well as Test 1 and 3. The value of the GDI for the right and left leg did not change significantly between the tests. Based on a literature review, we can state that, so far, no studies have investigated changes in gait pattern during nine months following a rehabilitation program in a large population of children with JIA. Hence, the current study is the first one to report scientific evidence related to this. Earlier research focused on evaluation of treatment effects in patients receiving corticosteroid injections into joints of lower limbs [11], [16] or on assessment of spatiotemporal gait parameters after outpatient physical therapy combined with a shockwave therapy session [17]. These studies reported varied results. For example, Esbjornsson et al. [16] did not observe improvement in spatiotemporal parameters or in the GDI either 3 weeks or 3 months after intra-articular corticosteroid injections. Conversely, Azab et al. [1] reported that, three months after outpatient physical therapy was applied to the affected knee in combination with a shock wave therapy, there was improvement in selected spatial parameters such as stance time and swing, velocity, cadence and step length.

The present study was not intended to assess the treatment applied and because of this, the effectiveness of interventions cannot be discussed. It is only clear that the baseline assessment was performed during a period of more severe problems, which is reflected by greater abnormalities in gait patterns; later the patients' overall improvement was accompanied with gradual normalization of their gait. Notably, however, they did not regain the gait pattern presented by their healthy peers.

The present findings confirm that despite significant advancements in treatment of children with JIA, as well as wider use of biologics and better availability of rehabilitation, patients with this disease continue to present gait deficits. When these abnormalities are detected and pathobiomechanics of gait is understood, it may be possible to adjust rehabilitation to patient's individual needs.

Further studies to assess the gait pattern in children with JIA should take into account symmetry. The ratio index and symmetry index of step length or stance phase may be an important assessment of the gait pattern and the effectiveness of the treatment used [3]. The study was also limited because information on the rehabilitation programme was collected only for the period between Test 2 and 3, and recreational and sports activities were not included. During the stay at the centre, the children and their parents received instructions on how to exercise at home and were informed about the possibilities of spending free time. At home, however, sports and rehabilitation activities were undertaken with different frequency, so it was not possible to carry out appropriate analyses.

\section{Conclusions}

The study showed that the gait pattern in children with JIA was significantly different regarding spatiotemporal and kinematic parameters, compared to that in healthy children. A rehabilitation program significantly improved the gait pattern in children with JIA, but differences in the gait pattern compared to healthy children were still present, and it means that an abnormal gait pattern in this group of patients became permanent.

\section{References}

[1] AZAB S.R., IBRAHIM A.R., RADWAN N.L., Effect of Extracorporeal Shockwave Therapy on Selected Gait Parameters in Children with Juvenile Idiopathic Arthritis, IJTRR., 2016, 5, 180-188.

[2] Beukelman T., Patkar N.M., SaAg K.G., Tolleson-Rinehart S., Cron R.Q., DeWitT E.M., Ilowite N.T., Kimura Y., LAXER R.M., LOVEll D.J., MARTINI A., Rabinovich C.E., Ruperto N., American College of Rheumatology Recommendations for the Treatment of Juvenile Idiopathic Arthritis: Initiation and Safety Monitoring of Therapeutic Agents for the Treatment of Arthritis and Systemic Features, Arthtitis Care Res., 2011, 4, 465-482.

[3] BŁażKiewicz M., Wiszomirska I., Wit A., Comparison of four methods of calculating the symmetry of spatial-temporal parameters of gait, Acta Bioeng Biomech., 2014, 16 (1), 29-35.

[4] Bohannon R.W., Comfortable and maximum walking speed of adults aged 20-79 years: reference values and determinants, Age Ageing., 1997, 26, 15-19. 
[5] Brostrom E., Hagelberg S., Haglund-Akerlind Y., Effect of joint injections in children with juvenile idiopathic arthritis: evaluation by 3D-gait analysis, Acta Paediatr., 2004, 93, 906-910.

[6] Brostrom E., Haglund-Akerlind Y., Hagelberg S., CRESSWELl A.G., Gait in children with juvenile chronic arthritis. Timing and force parameters, Scand J Rheumatol., 2002, 31, 317-323.

[7] Brostrom E., OrtQvist M., Haglund-Akerlind Y., Hagelberg S., Gutierrez-FarewiK E., Trunk and center of mass movements during gait in children with juvenile idiopathic arthritis, Hum. Mov. Sci., 2007, 26, 296-305.

[8] Davis R.B., Õunpuu S., Tyburski D., Gage J.R., A gait analysis data collection and reduction technique, Hum. Mov. Sci., 1991, 10, 575-587.

[9] EsbJörnsson A.C., Iversen M.D., André M., Hagelberg S., SchWARTZ M.H., BRoström E.W., Effect of Intraarticular Corticosteroid Foot Injections on Walking Function in Children With Juvenile Idiopathic Arthritis, Arthritis Care Res., 2015, 67, 1693-1701.

[10] Fairburn P.S., Panagamuwa B., Falkonakis A., Osborne S., Palmer R., Johnson B., Southwood T.R., The use of multidisciplinary assessment and scientific measurement in advanced juvenile idiopathic arthritis can categorise gait deviations to guide treatment, Arch. Dis. Child., 2002, 87, 160-165.

[11] Hartmann M., Kreuzpointner F., Haefner R., Michels H., SCHWIRTZ A., HAAS J.P., Effects of juvenile idiopathic arthritis on kinematics and kinetics of the lower extremities call for consequences in physical activities recommendations, Inter. J. Pediatr., 2010, 835984.

[12] Hartmann M., Schwirtz A., HäFner R., Michels H., Gait patterns of JIA polyarthritis - first kinematic results of a $3 D$ motion analysis, Aktuel Rheumatol., 2008, 33, 363-366.

[13] Hof L., Scaling gait data to body size, Gait Posture., 1996, 3 , 222-223.

[14] Houghton K.M., Guzman J., Evaluation of Static and Dynamic Postural Balance in Children With Juvenile Idiopathic Arthritis, Pediatr. Phys. Ther., 2013, 25, 150-157.

[15] JiNG-LonG H., New advances in Juvenile Idiopathic Arthritis, Chang Gung Med. J., 2012, 35, 1-14.

[16] KLEPPER S.E., Effects of an eight-week physical conditioning programme on disease signs and symptoms in children with chronic arthritis, Arthritis Care Res., 1999, 12, 52-60.

[17] Lechner E.D., McCarthy F.C., Holden M.K., Gait patterns in patients with juvenile rheumatoid arthritis, Phys Ther., 1987, 67, 1335-1341.
[18] Merker J., Hartmann M., HaAs J.P., Schwirtz A., Combined three-dimensional gait and plantar pressure analyses detecting significant functional deficits in children with juvenile idiopathic arthritis, Gait Posture, 2018, 66, 247-254.

[19] Merker J., Hartmann M., KreuzPointner F., Schwirtz A., HAAS J.P., Pathophysiology of juvenile idiopathic arthritis induced pes planovalgus in static and walking condition - A functional view using $3 d$ gait analysis, Pediatr. Rheumatol. Online J., 2015, 13, 21.

[20] Myer G.D., Brunner H.I., Melson P.G., Paterno M.V., FORD K.R., HEwETT T.E., Specialized Neuromuscular Training to Improve Neuromuscular Function and Biomechanics in a Patient With Quiescent Juvenile Rheumatoid Arthritis, Phys. Ther., 2005, 85, 791-802.

[21] Petty R.E., Southwood T.R., Manners P., Baum J., Glass D.N., GoldENBERG J., He X., MALdONADO-COCCO J., Orozco-Alcala J., Prieur A.M., Suarez-Almazor M.E., Woo P., International League of Associations for Rheumatology. International League of Associations for Rheumatology classification of juvenile idiopathic arthritis: second revision, Edmonton, 2001, J. Rheumatol., 2004, 31 (2), 390-392.

[22] RomickA A., Juvenile idiopathic arthritis - clinical picture, course of the disease and treatment, Med. Stand/Paediatr., 2010, 7, 189-193.

[23] Schwartz M.H., Rozumalski A., The Gait Deviation Index: a new comprehensive index of gait pathology, Gait Posture, 2008, 28, 351-357.

[24] TAKKen T., VAN Net J., Helders P.J., Do juvenile idiopathic arthritis patients benefit from exercise program? A pilot study, Arthritis Reum., 2001, 45, 81-85.

[25] Takken T., VAN Der Net J., Kuis W., Helders P.J.M., Aquatic fitness training for children with juvenile idiopathic arthritis, Rheumatology, 2003, 42(11), 1408-1414.

[26] Taracki E., Yeldan I., Baydogan N., Olgar S., KASAPCOPUR O., Efficiency of a land-based home exercise program for patients with juvenile idiopathic: A randomized controlled, single-blind study, J. Rehabil. Med., 2012, 44, 962-967.

[27] Van der Net J., Van der Torre P., Engelbert R.H., Engelen V., Van Zon F., TAKKen T., Helders P.J., Motor performance and functional ability in preschool-and early school-aged children with juvenile idiopathic arthritis: a cross-sectional study, Pediatr. Rheumatol. Online J., 2008, 6, 2. 